\title{
Long-term Follow-up of Patellar Nonresurfacing in Total Knee Arthroplasty
}

\author{
Young Choi, MD, Junsung Koo, MD, Sang Won Moon, MD*, Younghoon Yang, MD, Junghwan Son, MD \\ Department of Orthopaedic Surgery, Kosin University College of Medicine, Busan, \\ ${ }^{*}$ Department of Orthopaedic Surgery, Inje University College of Medicine, Haeundae Paik Hospital, Busan, Korea
}

\begin{abstract}
Background: We aimed to confirm the long-term effect of patellar nonresurfacing (patellar decompression) in preventing anterior knee pain after total knee arthroplasty (TKA) and to investigate the possible complications.

Methods: Among patients who underwent primary TKA after being diagnosed as having advanced osteoarthritis (Kellgren-Lawrence grade 4) at our institution from January 2004 to December 2010, 121 patients who were followed up for more than 7 years were included in this study. Patients who underwent TKA with and without patellar decompression were classified as the study group and control group, respectively. A clinical knee rating score was used to compare the postoperative clinical outcomes between groups. To identify complications after patellar decompression, simple radiographs (weight-bearing anteroposterior and lateral views, patella in $30^{\circ}$ and $45^{\circ}$ axial views, and whole scanogram) were taken during follow-up.

Results: There were no complications such as patellar fracture, osteonecrosis, and subluxation. At 2 years after surgery, the prevalence of anterior knee pain was $12.7 \%$ and $18.0 \%$ in the study group and control group, respectively $(p=0.42)$, and the number of patients with patellofemoral osteoarthritis grade II or over was lower in the study group $(p=0.03)$. At 7 years after surgery, the prevalence of anterior knee pain was $18.3 \%$ and $24.0 \%$ in the study group and control group, respectively $(p=0.45)$, and there was no statistically significant intergroup difference in the number of patients with patellofemoral osteoarthritis grade II or over (p $=0.11)$.
\end{abstract}

Conclusions: Patellar nonresurfacing TKA reduces anterior knee pain in the early postoperative period. The procedure can be considered a relatively safe option with fewer complications; however, its effectiveness appears to decrease over time.

Keywords: Knee, Osteoarthritis, Total knee replacement arthroplasty, Patellar decompression

Total knee arthroplasty (TKA) has been shown to be a successful procedure for treating advanced knee joint arthritis. Despite advances in techniques and implants, anterior knee pain is one of the most common problems after TKA. In the literature, anterior knee pain has been reported in $8 \%$ to $50 \%$ of cases after TKA. ${ }^{1-4)}$ Thus, after periprosthetic infection, patellofemoral joint problems are the second most common cause of revision TKA.-7)

Received May 23, 2019; Accepted August 13, 2019

Correspondence to: Junghwan Son, MD

Department of Orthopaedic Surgery, Kosin University Gospel Hospital,

262 Gamcheon-ro, Seo-gu, Busan 49267, Korea

Tel: +82-51-990-6467, Fax: +82-51-243-0181

E-mail: jhs@ns.kosinmed.or.kr
The causes of anterior knee pain can be divided into functional (muscle imbalance and dynamic valgus), structural (chondromalacia of the patella), mechanical (incorrect positioning of prosthetic components and aseptic loosening), and pathophysiologic (patellar hypertension syndrome) ${ }^{8-12)}$ Patellar resurfacing has been attempted to solve the structural problem, but the effect remains controversial. ${ }^{13-15)}$

The increase in intraosseous pressure of the distal femur causes anterior knee pain. ${ }^{12)}$ Several studies have reported on the pathophysiologic factors of anterior knee pain. ${ }^{16,17)}$ Since the introduction of patellar hypertension syndrome, new treatment concepts such as intraosseous drilling (patellar decompression) have been suggested. ${ }^{16}$ Several studies have reported on the short-term effects of 
Choi et al. Long-term Follow-up of Patellar Decompression

Clinics in Orthopedic Surgery • Vol. 12, No. 1, $2020 \bullet$ www.ecios.org

patellar decompression in anterior knee pain. ${ }^{9,16)}$ However, there are a limited number of studies on anterior knee pain after TKA with patellar hypertension. In this study, we aimed to confirm through long-term follow-up the effect of patellar decompression in preventing anterior knee pain after TKA without resurfacing and to investigate the possible complications.

\section{METHODS}

\section{Patients}

The study was conducted retrospectively by reviewing plain radiographs, medical records, and patient interviews. We conducted this study in compliance with the principles of the Declaration of Helsinki. The design and protocol of this retrospective study were approved by the Institutional Review Board of Kosin University Gospel Hospital (IRB No. 2019-04-020). Written informed consents were waived since this study was conducted retrospectively. Among patients who underwent primary TKA (Duracon; Howmedica, Rutherford, NJ, USA) after diagnosis of advanced osteoarthritis (Kellgren-Lawrence grade 4) at our institution from January 2004 to December 2010, 121 patients who were followed up for more than 7 years were included in this study. The exclusion criteria were history of systemic inflammatory disease, any disease causing lower extremity pain (e.g., herniated disc, spinal stenosis, and arteriosclerosis obliterans), and/or signs or symptoms of infection during follow-up. Patients who underwent TKA with and without patellar decompression were classified as the study group and control group, respectively. Patient data are shown in Table 1.

\section{Surgical Procedure}

All operations were done by a single surgeon (JS). All surgical approaches were performed with a midline skin inci-

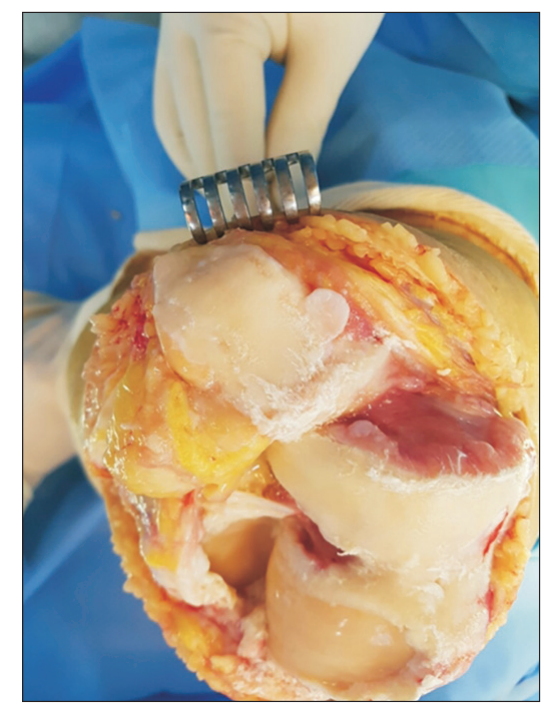

Fig. 1. Before patellar decompression, severe osteophytes and cartilage defect were identified.

\section{Table 1. Demographic Data}

\begin{tabular}{|lccc|}
\hline \multicolumn{1}{c}{ Variable } & Study group $(\mathrm{n}=71)$ & Control group $(\mathrm{n}=50)$ & $p$-value \\
\hline Age $(\mathrm{yr})$ & $69.9(62-81)$ & $70.5(60-83)$ & 0.53 \\
\hline Sex & & & 0.50 \\
\hline Male & $16(22.5)$ & $14(28.0)$ & \\
\hline Female & $55(77.5)$ & $36(72.0)$ & 0.16 \\
\hline Follow-up period $(\mathrm{mo})$ & $86.38(84-112)$ & $87.84(84-110)$ & 0.32 \\
\hline Body mass index $\left(\mathrm{kg} / \mathrm{m}^{2}\right)$ & $26.4(19.1-31.0)$ & $25.36(19.5-30.1)$ & 0.68 \\
\hline T-score (bone mineral density) & $-2.18 \pm 0.89$ & $-2.26 \pm 1.04$ & 0.72 \\
\hline Preoperative visual analog scale & $7.08 \pm 0.9$ & $7.16 \pm 1.29$ & 0.93 \\
\hline Preoperative osteoarthritis grade at the patellofemoral joint & & & $4(8.0)$ \\
\hline Grade I & $6(8.5)$ & $32(64.0)$ & 0.70 \\
\hline Grade II & $43(60.6)$ & $14(28.0)$ & 0.72 \\
\hline Grade III & $22(30.9)$ & & \\
\hline
\end{tabular}

Values are presented as median (range), number (\%), or mean \pm standard deviation. 
sion and mid-vastus approach (Fig. 1). The desired angle was $8^{\circ}$ valgus. A posterior cruciate ligament-retaining cemented implant was used in all patients (Duracon, Howmedica). Patellar resurfacing was not performed in any cases. In the study group, we first performed osteophyte removal of the patellar rim. Then, we used a $3.5-\mathrm{mm}$ drill to drill the patella via the fat pad under tissue protection in a parallel pattern (Fig. 2). Four to six holes were drilled parallel to the leg axis in the patella (Fig. 3).

\section{Evaluation}

We conducted a patient interview and used a simple clinical anterior knee pain rating to assess anterior knee pain. ${ }^{9)}$ The interview included following questions on the presence of pain, limitation of activity, and the need for additional surgery: (1) does anterior knee pain occur when rising from the chair? (2) Does anterior knee pain occur when ascending or descending the stairs? (3) Does anterior knee pain occur when squatting? (4) Does anterior knee pain occur when resting? (5) Is it difficult to fall asleep due to anterior knee pain? A clinical knee rating score was

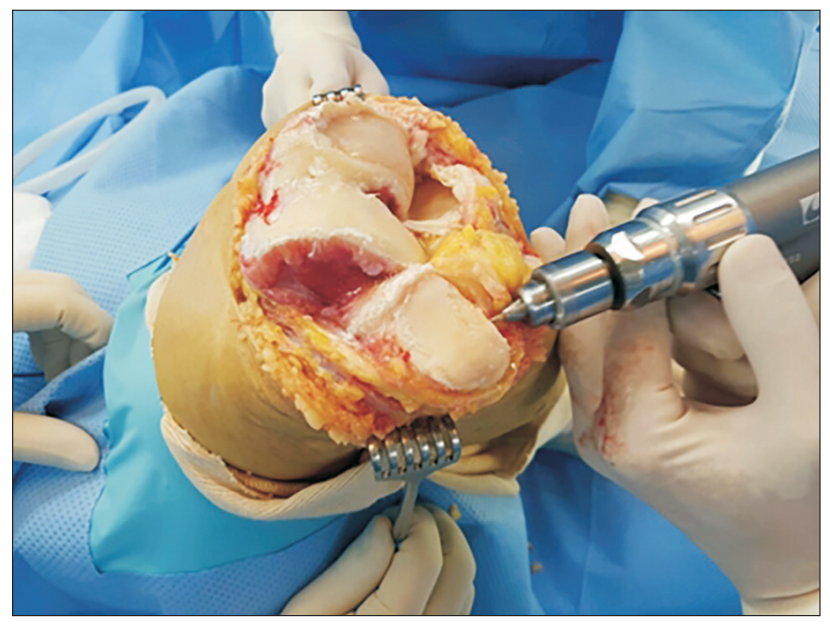

Fig. 2. We used a 3.5-mm drill to drill the patella via the fat pad under tissue protection in a parallel pattern. used to compare postoperative clinical outcomes. To identify complications after patellar decompression, simple radiographs (weight-bearing anteroposterior, lateral view, patella $30^{\circ}$ and $45^{\circ}$ axial views, and whole scanogram) were taken during follow-up.

\section{Statistical Analysis}

Statistical analysis was performed with IBM SPSS ver. 24.0 (IBM Corp., Armonk, NY, USA). Paired sample $t$-tests were used to compare preoperative and postoperative rates of the clinical knee rating score. Pearson's chi-square test was used to compare the anterior knee pain of the study and control groups. Student $t$-test was used to compare the demographic differences of the study and controls. A $p<$ 0.05 was considered statistically significant.

\section{RESULTS}

A total of 121 patients were followed up for more than 7 years after TKA. There were no statistically significant differences in demographics between the study and control groups (Table 1). Postoperative radiographs were assessed

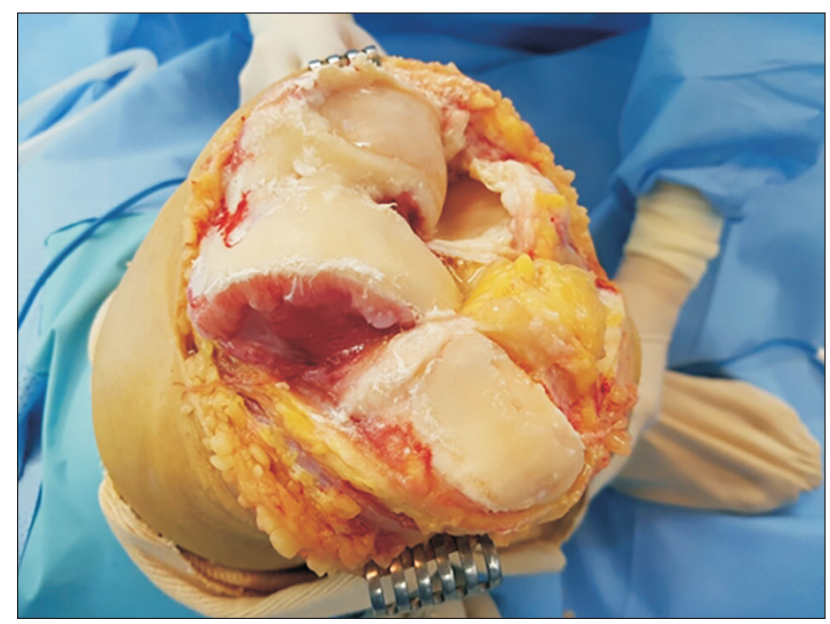

Fig. 3. Patellar decompression and osteophyte removal were performed.

Table 2. Anterior Knee Pain Rating at 2 Years after Surgery

\begin{tabular}{clcccc} 
Rating & \multicolumn{1}{c}{ Mean } & Visual analog scale & Study group $(\mathrm{n}=71)$ & Control group $(\mathrm{n}=50)$ & $p$-value \\
\hline & No pain & 0 & $62(87.3)$ & $41(82.0)$ & 0.418 \\
I & Mild pain & $1-3$ & $8(11.3)$ & $1(8.0)$ & 0.554 \\
II & Moderate pain & $4-6$ & $1(1.4)$ & $4(8.0)$ & 0.073 \\
III & Severe pain & $7-10$ & 0 & $1(2.0)$ & 0.231 \\
\hline
\end{tabular}

Values are presented as number (\%). 
for knee alignment, patellar tilt, and patellar height, and no significant differences were found between the groups. Follow-up radiography was performed every year until the last follow-up. There were no complications such as patellar fracture, osteonecrosis, and subluxation.

The prevalence of anterior knee pain was $12.7 \%$ in the study group and $18.0 \%$ in the control group at 2 years after surgery (Table 2 ), showing no statistically significant differences between groups $(p=0.42)$. However, the number of patients with patellofemoral osteoarthritis grade II or over was significantly lower in the study group $(p=$ 0.03 ). The prevalence of anterior knee pain was $18.3 \%$ in the study group and $24 \%$ in the control group at 7 years after surgery (Table 3 ), showing no statistically significant differences between groups $(p=0.45)$. The number of patients with patellofemoral osteoarthritis grade II or over was not statistically significantly different between groups at 7 years after surgery $(p=0.11)$.

The mean knee score improved from 43.25 points (range, 5 to 75 points) to 89.04 points (range, 70 to 100 points) in the study group and from 44.62 points (range, 10 to 75 points) to 85.24 points (range, 65 to 95 points) in the control group. The mean knee score was higher in the study group, but the difference was not statistically significant $(p=0.18)$. The postoperative mean knee function score was 76.42 points (range, 25 to 100 points) in the study group and 73.53 points (range, 20 to 100 points) in the control group, showing no statistically significant difference $(p=0.33)$.

Table 3. Anterior Knee Pain Rating at 7 Years after Surgery

\begin{tabular}{clcccc} 
Rating & \multicolumn{1}{c}{ Mean } & Visual analog scale & Study group $(\mathrm{n}=71)$ & Control group $(\mathrm{n}=50)$ & $p$-value \\
\hline 0 & No pain & 0 & $58(81.7)$ & $38(76.0)$ & 0.447 \\
I & Mild pain & $1-3$ & $10(14.1)$ & $2(12.0)$ & $5(10.0)$ \\
II & Moderate pain & $4-6$ & $1(1.4)$ & $1(2.0)$ & 0.739 \\
III & Severe pain & $7-10$ & 0.802 & 0.802 \\
\hline
\end{tabular}

Values are presented as number (\%).

\section{Table 4. Publications on Anterior Knee Pain after Total Knee Arthroplasty}

\begin{tabular}{|c|c|c|c|c|c|}
\hline Study & Type & No. of patients & Mean follow-up (mo) & Anterior knee pain & Functional score \\
\hline \multirow[t]{2}{*}{ Barrack et al. ${ }^{18)}$} & PR & 47 & 70.5 & $9(19.1)$ & - \\
\hline & NPT & 46 & 70.5 & $8(17.4)$ & \\
\hline \multirow[t]{2}{*}{ Waters and Bentley ${ }^{14)}$} & PR & 243 & $63.6(24-102)$ & $13(5.3)$ & \\
\hline & NPT & 231 & $64(2-102)$ & $58(25.1)$ & \\
\hline \multirow[t]{2}{*}{ Park et al. ${ }^{19)}$} & PR & 29 & $149(121-216)$ & & 77.5 \\
\hline & NPT & 44 & $140.7(122-168)$ & & 60 \\
\hline \multirow[t]{2}{*}{ Lee et al. ${ }^{9)}$} & PD & 124 & $60.8(54-86)$ & $23(18.5)$ & 90.1 \\
\hline & NPT & 131 & $62.2(48-87)$ & $28(21.3)$ & 86.74 \\
\hline \multirow[t]{4}{*}{ This study } & PD & 71 & $2 \mathrm{yr}$ & $9(12.7)$ & 76.4 \\
\hline & NPT & 50 & & $9(18.0)$ & \\
\hline & $\mathrm{PD}$ & 71 & $7 \mathrm{yr}$ & $14(18.3)$ & 73.5 \\
\hline & NPT & 50 & & $12(24.0)$ & \\
\hline
\end{tabular}

Values are presented as number (\%) or median (range).

PR: patellar resurfacing, NPT: neural prolotherapy, PD: patellar denervation. 
Choi et al. Long-term Follow-up of Patellar Decompression

Clinics in Orthopedic Surgery • Vol. 12, No. 1, $2020 \bullet$ www.ecios.org

\section{DISCUSSION}

The principle finding of this study is that patellar decompression performed by using a $3.5-\mathrm{mm}$ drill could reduce postoperative anterior knee pain in the early postoperative period, but the effect decreased in the long-term followup. Anterior knee pain after TKA is one of the most common complications. Many studies have focused on the structural characteristics to prevent this complication. Surgical methods suggested in these studies include patellar osteotomy, distal realignment procedure, and patellar resurfacing, but their effect remains controversial. ${ }^{13-15)} \mathrm{A}$ previous study conducted at our hospital introduced the concept of increased intraosseous pressure and patellar hypertension, which have been reported as one of the causes of anterior knee pain. ${ }^{9)}$ Some studies have reported that an impaired venous drainage induces intraosseous hypertension. ${ }^{16,17)}$ Schneider et al. ${ }^{16)}$ reported that if the venous pathway of the patella is impaired, patellar drilling leads to the immediate reduction of intraosseous pressure and pain relief.

In this study, we focused on the pathophysiologic factors of anterior knee pain and suggested patellar decompression as a solution. If anterior knee pain arises from patellar hypertension after TKA, it can be solved by patellar decompression. In the literature, anterior knee pain has been reported in $8 \%-50 \%$ of cases after TKA. ${ }^{1-4)}$ Patellar resurfacing is the most commonly used method, but the results are controversial and can lead to complications such as patellar replacement component wear, loosening, fractures, and osteonecrosis (Table 4). ${ }^{9,14,18,19)}$ In this study, there were no complications such as fracture or osteonecrosis after patellar decompression during follow-up, which is consistent with the findings of previous reports on patellar decompression. ${ }^{9)}$

The prevalence of anterior knee pain at two years after surgery was similar to that reported by Lee et al. ${ }^{9)}$ In both studies, compared to the group without patellar decompression, the group with patellar decompression had a greater number of patients with less than moderate pain at 2 years after surgery. However, in our study, there was no statistically significant intergroup difference in anterior knee pain at 7 years' follow-up. This is different from the result of Lee et al. ${ }^{9)}$ The reason for this difference may be attributable to the fact that the average follow-up period of Lee's study was approximately 60 months. On the basis of our 7-year follow-up results, we think that the effect of patellar decompression is maintained for up to 5 years, but the effect decreases thereafter.

This study has some limitations. First, there are few studies on the effect of patellar decompression; thus, comparison of the results was limited. However, patellar decompression is considered an effective technique in reducing anterior knee pain because it can be performed by drilling during primary TKA without the need for additional procedures. Second, because the intraosseous pressure was not directly measured in this study, there were some limitations in demonstrating the effectiveness of patellar decompression.

Patellar nonresurfacing in TKA can be performed during primary TKA without an additional incision. This procedure can reduce anterior knee pain in the early postoperative period. In conclusion, patellar nonresurfacing TKA can be a relatively safe and simple procedure with fewer complications, but its effect on relief of anterior knee pain appears to decrease in the long term.

\section{CONFLICT OF INTEREST}

No potential conflict of interest relevant to this article was reported.

\section{REFERENCES}

1. Sensi L, Buzzi R, Giron F, De Luca L, Aglietti P. Patellofemoral function after total knee arthroplasty: gender-related differences. J Arthroplasty. 2011;26(8):1475-80.

2. Ghomrawi HM, Schackman BR, Mushlin AI. Appropriateness criteria and elective procedures--total joint arthroplasty. N Engl J Med. 2012;367(26):2467-9.

3. Scott RD. The evolving incidence and reasons for re-operation after fixed-bearing PCL retaining total knee arthroplasty. J Bone Joint Surg Br. 2012;94(11 Suppl A):134-6.

4. Richmond JC. Surgery for osteoarthritis of the knee. Rheum
Dis Clin North Am. 2013;39(1):203-11.

5. Fehring TK, Odum S, Griffin WL, Mason JB, Nadaud M. Early failures in total knee arthroplasty. Clin Orthop Relat Res. 2001;(392):315-8.

6. Berger RA, Crossett LS, Jacobs JJ, Rubash HE. Malrotation causing patellofemoral complications after total knee arthroplasty. Clin Orthop Relat Res. 1998;(356):144-53.

7. Eisenhuth SA, Saleh KJ, Cui Q, Clark CR, Brown TE. Patellofemoral instability after total knee arthroplasty. Clin Orthop Relat Res. 2006;446:149-60. 
Choi et al. Long-term Follow-up of Patellar Decompression

Clinics in Orthopedic Surgery • Vol. 12, No. 1, $2020 \bullet$ www.ecios.org

8. Petersen W, Rembitzki IV, Bruggemann GP, et al. Anterior knee pain after total knee arthroplasty: a narrative review. Int Orthop. 2014;38(2):319-28.

9. Lee GW, Lee SM, Jang SJ, Son JH. The efficacy of patellar decompression for improving anterior knee pain following total knee arthroplasty without patellar resurfacing. Arch Orthop Trauma Surg. 2013;133(4):561-7.

10. Kim YM, Joo YB. Patellofemoral osteoarthritis. Knee Surg Relat Res. 2012;24(4):193-200.

11. Dye SF. Patellofemoral pain current concepts: an overview. Sports Med Arthrosc Rev. 2001;9(4):262-72.

12. Arnoldi CC, Lemperg K, Linderholm H. Intraosseous hypertension and pain in the knee. J Bone Joint Surg Br. 1975;57(3):360-3.

13. Wood DJ, Smith AJ, Collopy D, White B, Brankov B, Bulsara MK. Patellar resurfacing in total knee arthroplasty: a prospective, randomized trial. J Bone Joint Surg Am. 2002;84(2):187-93.

14. Waters TS, Bentley G. Patellar resurfacing in total knee arthroplasty: a prospective, randomized study. J Bone Joint
Surg Am. 2003;85(2):212-7.

15. Burnett RS, Haydon CM, Rorabeck CH, Bourne RB. Patella resurfacing versus nonresurfacing in total knee arthroplasty: results of a randomized controlled clinical trial at a minimum of 10 years' followup. Clin Orthop Relat Res. 2004;(428):12-25.

16. Schneider U, Breusch SJ, Thomsen M, Wenz W, Graf J, Niethard FU. A new concept in the treatment of anterior knee pain: patellar hypertension syndrome. Orthopedics. 2000;23(6):581-6.

17. Heyse TJ, Becher C, Kron N, et al. Patellofemoral pressure after TKA in vitro: highly conforming vs. posterior stabilized inlays. Arch Orthop Trauma Surg. 2010;130(2):191-6.

18. Barrack RL, Bertot AJ, Wolfe MW, Waldman DA, Milicic M, Myers L. Patellar resurfacing in total knee arthroplasty: a prospective, randomized, double-blind study with five to seven years of follow-up. J Bone Joint Surg Am. 2001;83(9):137681.

19. Park SJ, Jung YB, Jeong HJ, et al. Long-term results of primary total knee arthroplasty with and without patellar resurfacing. Acta Med Okayama. 2010;64(5):331-8. 